\title{
Intervenção da Terapia Ocupacional no desempenho ocupacional na sexualidade de pessoas com lesão medular: relato de caso
}

\author{
Intervención de Terapia Ocupacional en el desempeño ocupacional \\ de la sexualidad en personas con lesión medular: reporte de caso \\ Intervention of Occupational Therapy in occupational performance \\ of sexuality in persons with spinal cord injury: case report
}

\author{
Simone De Almeida ${ }^{7}$ \\ Raphael Fabricio De Souza² \\ Aristela De Freitas Zanona ${ }^{3}$
}

Recibido: 6 de septiembre 2017 • Enviado para modificación: 14 de noviembre 2017 • Aceptado: 25 de febrero 2018

De Almeida, S., De Souza, R.F. \& Zanona, A.F. (2018). Intervenção da Terapia Ocupacional no desempenho

ocupacional na sexualidade de pessoas com lesão medular: relato de caso. Revista Ocupación Humana, 18(1), 50-64.

\section{RESUMO}

A sexualidade compreende um processo complexo e natural, no entanto, após a lesão da medula espinhal vários problemas físicos, emocionais e sociais podem comprometer o desempenho satisfatório da atividade sexual. O objetivo desta pesquisa foi identificar os efeitos de um programa terapêutico ocupacional sobre a sexualidade de dois sujeitos de sexo masculino com lesão medular. As variáveis selecionadas foram: qualidade de vida, desempenho ocupacional e função sexual. O protocolo de intervenção consistiu na educação e discussão sobre sexualidade e lesões da medula espinhal; exercícios para fortalecer membros superiores, tronco e assoalho pélvico; simulações de posições sexuais consideradas difíceis de executar de acordo com as condições motoras dos sujeitos. A investigação foi realizada

\footnotetext{
${ }^{1}$ Estudiante de Terapia Ocupacional, Universidade Federal de Sergipe. Lagarto, Brasil simonealmeidabrito@gmail.com iD https://orcid.org/0000-0002-9160-2132

${ }^{2}$ Licenciado en Educación Física. Magíster en Educación Física. Doctorando en Neuropsiquiatría y Ciencias del Comportamiento, Universidade Federal de Pernambuco. Docente curso de Educação Física, Universidade Federal de Sergipe. São Cristóvão, Brasil. raphaelctba20@hotmail.com (iD) https://orcid.org/0000-0003-0684-2092

${ }^{3}$ Terapeuta ocupacional. Magíster en Educación Física. Doctoranda en Neuropsiquiatría y Ciencias del Comportamiento, Universidade Federal de Pernambuco. Docente curso de Terapia Ocupacional, Universidade Federal de Sergipe. Lagarto, Brasil. arisz_to@yahoo.com.br iD https://orcid.org/00000002-3899-6849
} 
entre janeiro e março de 2017. Para a análise dos resultados, foram utilizadas estatísticas descritivas com medidas de tendência central, delta e porcentagem para avaliar pacientes na pré e pós-intervenção. Houve uma melhoria significativa na qualidade de vida, desempenho ocupacional e função sexual após a intervenção da Terapia Ocupacional.

\title{
PALAVRAS CHAVE
}

traumatismos da medula espinhal, Terapia Ocupacional, sexualidade, qualidade de vida

\section{RESUMEN}

La sexualidad comprende un proceso complejo y natural, sin embargo, después de una lesión medular, diversos problemas de orden físico, emocional y social pueden comprometer el desempeño satisfactorio de la actividad sexual. En este artículo se reportan los efectos de un programa terapéutico ocupacional sobre la sexualidad de dos sujetos de sexo masculino con lesión medular, teniendo en cuenta las variables: calidad de vida, desempeño ocupacional y función sexual. El protocolo de intervención consistió en educación en salud y discusión sobre la lesión medular y la sexualidad; ejercicios de fortalecimiento de miembros superiores, tronco y piso pélvico; simulaciones de posiciones sexuales consideradas difíciles de ejecutar, de acuerdo con las condiciones motoras de los sujetos. La investigación transcurrió entre enero y marzo de 2017. Para el análisis de los resultados se utilizó estadística descriptiva con medidas de tendencia central, delta y porcentaje para evaluar a los pacientes en el pre y post intervención. Se verificó una mejora significativa en la calidad de vida, en el desempeño ocupacional y en la función sexual después de la intervención de Terapia Ocupacional.

\section{PALABRAS CLAVE}

traumatismos de la médula espinal, Terapia Ocupacional, sexualidad, calidad de vida

\begin{abstract}
Sexuality is a broad and complex process, natural to individuals but, after a spinal cord injury, various physical, emotional and social problems can compromise the satisfactory performance of sexual activity. This article reports the effects of an occupational therapeutic program on the sexuality of two individuals with spinal cord injury, based on quality of life, occupational performance and sexual function. The intervention protocol consisted of health education and discussion on Spinal Cord Injury and sexuality; exercises to strengthen the upper limbs, core and pelvic floor. Simulations of sexual positions considered difficult to execute were also carried out. The research was carried out between January and March 2017. For the analysis of the results, descriptive statistics were used with measures of central tendency, delta and percentage to evaluate the patients in the pre and post intervention. There was a significant improvement in quality of life, in the occupational performance and in the sexual function after the Occupational therapy intervention.
\end{abstract}

\section{KEY WORDS}

spinal cord injury, Occupational Therapy, sexuality, quality of life 


\section{Introdução}

A lesão medular é um evento inesperado, caracterizado pela interrupção total ou parcial do sinal neurológico através da medula espinhal. Tal fato, resulta em diminuição ou ausência das funções corporais da motricidade e sensibilidade, assim como distúrbios neurovegetativos corporais situados abaixo do nível da lesão (Campos, Ribeiro, Listik, Pereira, Sobrinho \& Rapoport, 2008; Rieder, 2014).

A lesão medular pode ser ocasionada por traumas primários e secundários. A lesão primária é ocasionada pela transferência de energia cinética para a medula espinhal, seguida por rompimento dos axônios, afetando as células nervosas e ocasionando danos aos vasos sanguíneos. Deste modo, a lesão primária resulta do trauma mecânico, provocado pelo impacto inicial seguido por uma compressão. $\mathrm{O}$ dano é possível acontecer de quatro formas: estiramento, laceração, compressão e secção; de acordo com o mecanismo da lesão, os danos nos axônios, células gliais e nos vasos sanguíneos, podem sofrer comprometimentos em diferentes graus. Já a lesão secundária acontece pela isquemia pós-traumática, provocada por infarto da medula espinhal, hipóxia e desvios iônicos intracelulares, excitoxicidade neuronal, produção de radicais livres, peroxidação de lipídios, apoptose neuronal, desacoplamento da produção de energia e do metabolismo celular (Rieder, 2014).

Em sua maioria, a lesão medular é provocada por acidentes automobilísticos e por armas de fogo. A maioria das vítimas são do sexo masculino e em idade produtiva. Os casos de lesão medular em idosos e em crianças são provenientes de quedas, em sua pluralidade. Também foram notificados casos de lesão medular relacionados à presença de tumores, mielomenigocele, prática de esportes, e à violência corporal (Baasch, 2008). A incidência global dessa patologia é mais prevalente em áreas urbanas (Silva, 2015). No Brasil, são estimados cerca de 6 a 8 mil novos casos por ano (Morais et al., 2013).

Quanto mais alto o nível da lesão, maior será o comprometimento neurológico, sensitivo e motor do corpo e as consequências nas habilidades práxico-motoras e percepto-sensoriais (Scussel, 2016).

A lesão acima de C7 compromete membros superiores, inferiores, tronco e funções dos órgãos internos e a lesão abaixo de T1 pode comprometer o tronco, os membros inferiores, sistema geniturinário e reprodutivo. A lesão que rompe totalmente a circuitaria comunicante, entre medula e cérebro, é chamada de lesão completa. Já quando a comunicação é descontinuada parcialmente atribui-se o nome lesão incompleta (Borges, Brignol, Schoeller \& Bonetti, 2012).

Dentre os sistemas comprometidos, os sistemas urinário, respiratório, circulatório, intestinal, reprodutivo e sexual podem sofrer danos que, dependendo do nível da lesão, são irreversíveis. Isso ocorre porque as vias aferentes e eferentes, envolvidas nesse percurso neurológico, são danificadas, resultando na alteração ou perda da função motora, sensibilidade, no controle do vaso motor, da bexiga, do intestino e da função sexual (Scussel, 2016). 
Em condições normais, as atividades sexuais seguem um processo natural, controlado pelo Sistema Nervoso Central que envia as mensagens à medula espinhal, provocando reações no sistema autônomo (como a ereção) (Aquarone, Faro \& Nogueira, 2015). Para que essas reações aconteçam, os neurotransmissores dopaminérgicos são disparados na fenda sináptica, despolarizando a membrana, sendo considerado um potencial de ação excitatório do sistema nervoso. Essa estimulação vinda do Sistema Nervoso Central é chamada de psicogênica e ativa o comando sacral. Porém, a estimulação reflexa também é possível mediante o comando presente no circuito sacral S2, S3, S4. No homem, essa reação é provocada pelo estímulo psicogênico ou pelo reflexo, provoca a vasoconstrição peniana, enchendo os corpos cavernosos e, consequentemente, garantindo uma ereção mais firme e duradoura. Nas mulheres, o circuito sacral emitirá sinais para que a vagina sofra dilatação e lubrificação, permitindo todo o processo sexual, entretanto, quando esse percurso é interrompido, o indivíduo precisará encontrar formas de vivenciar a sexualidade. A partir de então, é primordial que a equipe de saúde esteja apta para promover os esclarecimentos da existência de novas formas de exploração da sexualidade (Maia, 2012).

De acordo com o tipo de lesão medular, a estimulação reflexa (ou psicogênica) pode estar presente. Dessa forma, a ereção peniana e a lubrificação vaginal, podem estar ativas, embora menos efetivas, comparadas ao período anterior à lesão. No caso da lesão incompleta é possível que raízes nervosas, responsáveis pela estimulação reflexa, estejam íntegras e, por isso, sua conexão ainda responsiva. A lesão entre os seguimentos T2 e S2 apresenta os centros simpáticos e parassimpáticos íntegros, permitindo a ereção reflexa e psicogênica, a qual se manifesta por estímulos sensoriais, mas mantém-se por tempo não satisfatório (Torrecilha, Costa, Lima, Santos \& Souza, 2014).

As alterações orgânicas não devem inibir a continuidade das atividades inerentes ao sujeito, tais como a sexualidade. O ato sexual é definido como algo relacionado à fisiologia, contudo, a sexualidade representa o ser humano em sua plenitude. Dessa forma, a sexualidade não é perdida em consequência de uma doença (Nepomuceno, Melo \& Silva, 2014).

A sexualidade compreende um processo amplo e complexo, natural aos indivíduos, que se estende muito além do comportamento biológico (Maia, 2012). Portanto, a sexualidade não se restringe aos órgãos genitais, porque qualquer região do corpo está apta a sentir prazer, desde que ocorra investimento de erotismo (Barbosa, 2003). No entanto, quando se fala em sexualidade, costuma-se remeter ao contato restrito ao genital, priorizando o orgasmo, decorrente de penetração. O contato inicial, com a presença de carícias e toques, é promotor de excitação e, assim, torna o sexo apenas uma das facetas da sexualidade (Baasch, 2008). Esse entendimento é necessário aos indivíduos, a fim de fazê-los conhecer o próprio corpo, os pontos de excitação, bem como reaprender a agradar a si e ao parceiro (Barbosa, 2003).

Os profissionais de saúde devem enfocar essa questão como parte do 
planejamento terapêutico de cada paciente, afim de possibilitar a interação do sujeito em todos os seus contextos. Já que a sexualidade faz parte da área de desempenho ocupacional humano, cabe ao terapeuta ocupacional facilitar as conexões afetivas e como consequência a inclusão desse paciente no meio social de forma plena, com acesso aos diversos contextos que fazem parte de sua vida. O entendimento a respeito desses valores são indispensáveis para o retorno saudável às suas atividades diárias (Carleto, Cavalcanti, Souza, Cruz \& Andrade, 2010).

Desse modo, a Terapia Ocupacional como agente de promoção de saúde, entende que a sexualidade é um componente indispensável ao ser humano por significar um agente equilibrador da qualidade de vida juntamente com as demais áreas de desempenho. Cabe aos profissionais de reabilitação, em especial ao terapeuta ocupacional, fornecer conhecimentos referentes à atividade sexual, construindo, em parceria, uma estratégia de solução para o problema, procurando orientar além do limite do atendimento individual, abrangendo os parceiros e familiares, possibilitando, assim, de forma eficaz, a realização pessoal do indivíduo (Carleto et al., 2010).

Diante do exposto, este trabalho tem como objetivo identificar os efeitos de um programa terapêutico ocupacional sobre a sexualidade de dois sujeitos com lesão medular.

\section{Materiais e Método}

A pesquisa consiste em um reporte de caso, do tipo exploratório, com caráter quantitativo e delineamento longitudinal. Inicialmente, a amostra foi composta por pacientes com idade entre 18 e 40 anos, do sexo masculino, com diagnóstico confirmado de lesão medular, mediante Classificação Internacional de Doenças CID específica.

Como critérios de elegibilidade, foram incluídos pacientes com diagnóstico de lesão medular há pelo menos seis meses, podendo ser lesão completa ou incompleta; sem problemas de audição, visão ou fala; e não estar recebendo atendimentos de reabilitação (fisioterapia, terapia ocupacional) durante o evento da pesquisa; a fim de evitar variável de confusão nos resultados do protocolo desta pesquisa. Foram excluídos aqueles que apresentaram diagnóstico de doenças mentais, déficits neurológicos (diferentes da lesão medular) ou déficits cognitivos, pessoas que se recusassem a participar da pesquisa, mesmo havendo assinado o TCLE; os que não conseguiram os meios de locomoção necessários para chegar à clínica; sujeitos em fase aguda da lesão medular ou com menos de seis meses de lesão.

A amostra inicial desta pesquisa consistiu em doze sujeitos, contudo, sete participantes foram excluídos do estudo devido às dificuldades de transporte; outros quatro sujeitos, devido à comprometimentos secundários, tais como úlceras de decúbito; e um outro, com comorbidades associadas (amputação de membro inferior). Assim, apenas dois sujeitos responderam aos critérios de inclusão e participaram do estudo.

\section{Desfecho e medidas de desfecho}

Elegeu-se como desfecho primário 
desta pesquisa a função sexual de indivíduos com lesão medular. Os desfechos secundários elencados são qualidade de vida e desempenho ocupacional.

Para avaliação da função sexual, foi utilizado o Quociente Sexual Masculino - QSM (Abdo, 2006). O Quociente Sexual Masculino -QSM pode ser interpretado em escores totais, verificando a qualidade e satisfação sexual. O questionário é composto de dez questões, de caráter auto responsivo contemplando os domínios: desejo, interesse sexual, preliminares, excitação pessoal, sintonia com o parceiro, conforto, orgasmo e satisfação. Deve ser respondido numa escala que varia de 0 a 5 . O escore obtido é multiplicado por 2, resultando em valor que pode variar entre 0 a 100, com valores maiores, indicando melhor desempenho e satisfação sexual.

A qualidade de vida foi avaliada com o propósito de verificar a percepção do indivíduo acerca de seu bem estar geral. $\mathrm{O}$ instrumento utilizado foi a versão brasileira do questionário de qualidade de vida: The Short Form (36) Health Survey, conhecido por SF-36 (Ciconelli, Ferraz, Santos, Meinao \& Quaresma, 1999). Este instrumento aborda 36 itens que investigam a percepção dos indivíduos sobre a própria saúde nas últimas quatro semanas. As questões são divididas em oito domínios: capacidade funcional, limitação por aspectos físicos, dor, estado geral de saúde, vitalidade, aspectos sociais, aspectos emocionais e saúde mental. Para cada domínio, o escore variou de 0 a 100, em que, quanto mais próximo de zero, pior é a qualidade de vida, e quanto mais próximo de 100 , melhor a qualidade de vida correspondente àquele domínio.
A variável "desempenho ocupacional" foi analisada através da aplicação da Medida Canadense de Desempenho Ocupacional - COPM (Law et al., 2009). Trata-se de uma medida individual do sujeito acerca de sua autopercepção dos problemas encontrados no seu próprio cuidado (tais como se vestir, tomar banho, se alimentar, atividade sexual, etc), atividades instrumentais de vida diária (mobilidade na comunidade, fazer compras, etc), educação, trabalho e lazer. Os escores atribuídos de 1 a 10 indicam que, quanto menor o escore, pior é o desempenho e a satisfação com a execução daquela tarefa no dia a dia.

\section{Descrição da intervenção}

Os sujeitos foram submetidos às avaliações e às reavaliações com os instrumentos supracitados, aplicados pela mesma pesquisadora, sob a forma de questionários durante a entrevista. A intervenção foi realizada em sessões individuais com frequência semanal (duas vezes na semana), de fevereiro a março de 2017, totalizando 10 atendimentos, com duração de cinquenta minutos cada.

Após a análise da literatura e o levantamento das principais queixas dos sujeitos, o protocolo elaborado consistiu em atividades de educação em saúde, a respeito da sexualidade e sua função no desempenho de papéis ocupacionais do indivíduo; exercícios de fortalecimento de membros superiores, tronco e assoalho pélvico foram executados, a fim de maximizar as habilidades para assumir e manter posturas durante a atividade sexual. Também foram realizadas simulações de posições sexuais consideradas difíceis de executar pelos pacientes. Ademais, dinâmicas, cujo o objetivo era de favorecer a 
autoestima e o bem-estar geral, foram executadas.

\section{Análise estatística}

Para a análise dos resultados, foi utilizada estatística descritiva com medidas de tendência central (média), delta (subtração do valor máximo pelo mínimo) e porcentagem para avaliar os pacientes na pré e pós intervenção. Foi utilizado o programa Excel da Microsoft ${ }^{\oplus}$, Windows 8.

\section{Considerações éticas}

Este estudo foi aprovado pelo Comitê de Ética em Pesquisa do Hospital Universitário da Universidade Federal de
Sergipe (CAAE 62176016.2.0000.5546). Os participantes e os responsáveis pelas instituições foram informados sobre os objetivos e procedimentos do estudo.

Os participantes assinaram o Termo de Consentimento Livre e Esclarecido, conforme resolução $n^{\circ}$ 466/12 do Conselho Nacional de Saúde.

\section{Resultados}

\section{Caracterização}

As informações de caracterização dos dois participantes do estudo estão na tabela 1.

Tabela 1. Caracterização dos participantes

\begin{tabular}{|lll|}
\hline & Participante I & Participante II \\
\hline Idade & 35 & 33 \\
\hline Gênero & $\mathrm{M}$ & $\mathrm{M}$ \\
\hline Orientação sexual & Heterossexual & Heterossexual \\
\hline Estado civil & Casado & Casado \\
\hline Escolaridade & $1^{\circ}$ grau completo & Ensino superior incompleto \\
\hline Nível de lesão & Lesão T11-T12, lesão completa & L1-L2 incompleta \\
\hline Tempo de lesão & 2 anos e 8 meses & 2 anos e 7 meses \\
\hline
\end{tabular}

Fonte: Elaboração própria

\section{Função sexual}

Com relação a função sexual, houve melhora considerada apenas por um participante, identificada nos domínios do Quociente Sexual Masculino
- QSM: desejo, conforto e satisfação sexual (Abdo, 2006). O cálculo realizado levou em conta o escore da avaliação pré e pós intervenção. Os resultados estão representados na tabela 2 . 
Tabela 2. Quantificação do Questionário Sexual Masculino - QSM

\begin{tabular}{|c|c|c|c|}
\hline Identificação & Pré-Intervenção & Pós-Intervenção & $\Delta \%$ \\
\hline Participante I & 46 & 46 & - \\
\hline Participante II & 72 & 80 & $8 \%$ \\
\hline
\end{tabular}

$\Delta \%=$ Percentual de mudança do delta

Fonte: Elaboração própria

\section{Desempenho ocupacional}

Para a variável desempenho ocupacional, avaliada pela Medida Canadense de Desempenho Ocupacional - COPM (Law et al., 2005) foi possível identificar melhora na percepção dos sujeitos tanto no desempenho ocupacional quanto na satisfação nas áreas de autocuidado: vestir-se com independência, tomar banho, mobilidade e vida sexual. Os resultados estão representados na tabela 3 .

Tabela 3. Resultado do Questionário da Medida

Canadense de Desempenho Ocupacional - COPM

\begin{tabular}{|c|c|c|c|c|c|c|}
\hline \multirow{2}{*}{ Identificação } & \multicolumn{2}{|c|}{ Pré-Intervenção } & \multicolumn{2}{|c|}{ Pós-Intervenção } & \multicolumn{2}{|c|}{$\Delta \%$} \\
\hline & D & $\mathbf{S}$ & D & $\mathbf{S}$ & D & $\mathbf{S}$ \\
\hline Participante I & 4,0 & 6,0 & 8,0 & 8,0 & $40 \%$ & $20 \%$ \\
\hline Participante II & 5,0 & 4,0 & 8,0 & 8,0 & $30 \%$ & $40 \%$ \\
\hline
\end{tabular}

$\mathrm{D}=$ Desempenho, $\mathrm{S}=$ Satisfação, $\Delta \%=$ Percentual de mudança do delta.

Fonte: Elaboração própria 


\section{Qualidade de vida}

Com relação à Qualidade de vida, ambos os participantes apresentaram melhora em todos os domínios do Questionário de Qualidade de Vida SF-36, destacando-se diminuição da limitação por aspectos físicos e aspecto emocional. Os resultados totais de todos os domínios estão na tabela 4, com percentual de mudança representado pelo delta entre escores pós e pré intervenção.

Tabela 4. Resultado da avaliação do Questionário de Qualidade de Vida - SF-36

\begin{tabular}{|c|c|c|c|c|c|c|}
\hline \multirow[t]{2}{*}{ Identificação } & \multicolumn{2}{|c|}{ Participante I } & \multirow[t]{2}{*}{$\Delta \%$} & \multicolumn{2}{|c|}{ Participante II } & \multirow[t]{2}{*}{$\Delta \%$} \\
\hline & Pré & Pós & & Pré & Pós & \\
\hline $\begin{array}{l}\text { Capacidade } \\
\text { funcional }\end{array}$ & 55 & 65 & $10 \%$ & 45 & 55 & $10 \%$ \\
\hline $\begin{array}{l}\text { Limitação } \\
\text { aspectos físicos }\end{array}$ & 0 & 100 & $100 \%$ & 50 & 100 & $50 \%$ \\
\hline Dor & 51 & 84 & $33 \%$ & 51 & 51 & $\mathbf{0} \%$ \\
\hline $\begin{array}{l}\text { Estado geral de } \\
\text { saúde }\end{array}$ & 72 & 80 & $08 \%$ & 52 & 62 & $10 \%$ \\
\hline Vitalidade & 65 & 70 & $5 \%$ & 40 & 60 & $20 \%$ \\
\hline $\begin{array}{l}\text { Aspectos } \\
\text { sociais }\end{array}$ & 75 & 87 & $12 \%$ & 75 & 87 & $12 \%$ \\
\hline $\begin{array}{l}\text { Aspecto } \\
\text { emocional }\end{array}$ & 0 & 100 & $100 \%$ & 100 & 100 & $0 \%$ \\
\hline Saúde mental & 52 & 72 & $20 \%$ & 64 & 76 & $12 \%$ \\
\hline
\end{tabular}

$\Delta \%=$ Percentual de mudança do delta

Fonte: Elaboração própria

\section{Discussão}

A etiologia da lesão medular está, em sua maioria, ligada às causas traumáticas, provocadas por acidente automobilístico ou ferimentos por arma de fogo. Tais evidências foram abordadas nos trabalhos de Kawanishi \& Greguol (2014) e Silva et al. (2015), em que os estudos apontam como causa o aumento de acidentes com veículos automotores, sugerindo também que a população jovem, do sexo masculino, está mais exposta a acometimento por traumas. O acometimento por lesão medular na faixa etária produtiva im- plica em grave problema financeiro ao erário, visto que tal circunstância limita a capacidade funcional do indivíduo, provocando dificuldades sociais e econômicas tanto para o sujeito quanto para a sociedade (Kawanishi \& Greguol, 2014). Os resultados de ambos os participantes corroboram a literatura sobre a correlação entre indivíduos que sofreram lesão medular, gênero e idade.

Com relação ao desempenho ocupacional, a independência funcional de indivíduos com lesão medular pode ser gravemente afetada, pois, muitas 
vezes, estes acabam sofrendo uma ruptura de seus papéis ocupacionais, interferindo diretamente em suas relações pessoais e sociais. Devido às sequelas em diversos componentes, o sujeito apresenta prejuízo em sua autoestima, além do fator dependência de terceiros para execução de atividades cotidianas (Silva, 2015; Alves \& Duarte, 2010). Esta relação de dependência de terceiros, que comumente é o cônjuge, pode afetar significativamente o modo como o casal se relaciona intimamente.

Com o intuito de fornecer conhecimento acerca do desempenho sexual, foi apresentado, em forma de aula expositiva, a sexualidade humana, a fim de facilitar a compreensão da temática e ajudar a traçar planos e estratégias reais de enfrentamento da nova condição. Desse modo, foi verificado que, antes das intervenções, o conhecimento que os participantes possuíam acerca de sua sexualidade, estavam restritos ao ato sexual, desconsiderando suas possibilidades, que vão muito além do contato genital. A forma que o sujeito vivencia sua condição influencia diretamente na maneira de enxergar a sua imagem corporal e, certamente, impacta na qualidade de vida (Lima, Torres \& Filho, 2014).

Nenhuma característica, seja esta relacionada à idade ou à deficiência, impossibilita o indivíduo de exercer a sexualidade. Porém, as pessoas com algum tipo de deficiência, por muito tempo foram vistas como sujeitos "santificados" e que, portanto, não tinham possibilidade de vivenciar a sexualidade como inerente ao ser humano. O exercício da sexualidade culmina em satisfação pessoal, proveniente das emoções, que resultam em uma série de acontecimentos que geram qualidade de vida (Nepomuceno, Melo \& Silva, 2014).

Além das atividades de educação em saúde, exercícios de fortalecimento foram inseridos no protocolo, pois é sabido que o trabalho de fortalecimento perineal e global pode melhorar, com certa expressão, o desempenho físico durante o ato sexual, por promover maximização da função muscular. Isso facilita a contração dos músculos do períneo atuantes no mecanismo da ereção e, consequentemente, possibilita maior sensação prazerosa (Medeiros, Braz \& Brongholi, 2004). O aumento da força muscular de membros superiores e do tronco favoreceu para que ambos os participantes conseguissem executar, de maneira mais efetiva, trocas posturais não só durante o desempenho sexual, mas também foi possível perceber a generalização do aumento de força que contribuiu em outras atividades do cotidiano (Gonzáles \& Sties, 2015).

Com relação à função sexual, o escore do Quociente Sexual Masculino - QSM indicou pouca (participante II) ou nenhuma (participante I) mudança, isso, segundo Silva et al. (2017), pode acontecer pelo fato de os participantes não terem oportunidade de testar seu desempenho sexual durante e após as intervenções. Tal fato foi observado no presente estudo, o que, de certa forma, pode ter influenciado os resultados apresentados. A impossibilidade de executar o que foi aprendido com um parceiro pode indicar um prejuízo na composição dos papéis familiares. Ambos os participantes identificaram problemas conjugais após o evento da lesão medular. O casamento sofre enorme desgaste, pois não somente o paciente, mas tam- 
bém o parceiro, precisam passar pela fase de readaptação, em que são necessários reajustes do equilíbrio emocional (Torrecilha et al., 2014).

A relação vivida pelo casal tende a tomar características mais maternais, o que prejudica enormemente os desejos que ambos sentiam antes um pelo outro. A sexualidade vivenciada, após a lesão medular, demonstrou maior satisfação quando o sujeito e a família aceitam a nova condição, e se abrem para as novas perspectivas e conquistas.

Após a intervenção, foi notório o aumento dos escores do Questionário de Qualidade de Vida - SF-36, indicando que houve melhora na qualidade de vida. A lesão medular é uma das condições de saúde responsável por uma restrição do cotidiano, interferindo nos aspectos biológicos e psicológicos que limitam o estilo de vida, ocasionando a necessidade de adaptação (Silva et al., 2017). No domínio capacidade funcional, compreendido como habilidades para subir escadas, andar um quilômetro, varrer a casa, passar aspirador de pó, dentre outros, foi possível identificar aumento dos escores, indicando diminuição do cuidado de terceiros. Embora os treinos de atividades de vida diária e atividades instrumentais de vida diária, não foram diretamente executados, pode-se inferir que a melhora em habilidades práxico-motoras, tais como ganho de força muscular, aprendizado de trocas posturais e maior controle dos movimentos, podem ter impactado positivamente as áreas de ocupação correspondentes ao cuidado próprio e do ambiente domiciliar.

O domínio "limitação por aspectos físicos" indicou que piores condições físicas interferem na redução do tempo dedicado ao trabalho e outras atividades, realizando menos tarefas com necessidade de esforço extra para concluí-las. Os aspectos físicos podem incluir força, destreza, coordenação, resistência, sensibilidade, capacidade cardiorrespiratória, dentre outras (Silva, 2015). Após a intervenção, houve diminuição da limitação física e aumento da participação em atividades significativas.

Com relação ao domínio dor houve melhora nos escores. A dor pode exercer um papel bastante negativo, não só no desempenho da sexualidade, como no desempenho ocupacional geral (Aquarone, Faro \& Nogueira, 2015). A atividade física, como o fortalecimento, libera endorfina, potente analgésico endógeno que reduz a dor e o estresse e, além disso, aumenta a produção de serotonina. A serotonina é um neurotransmissor que possui forte efeito no humor, memória e aprendizado, regula o equilíbrio corporal e produção de noradrenalina, outro neurotransmissor que induz a excitação física, mental e bom humor. Todos esses neurotransmissores inibem quadros dolorosos, além de proporcionar sensação de bem estar (Tatar, 2010). Desta forma, acredita-se que as os exercícios executados podem ter diminuído a dor corporal geral.

Com relação aos domínios "estado geral de saúde" e "vitalidade", as intervenções utilizadas podem ter correlação positiva com a maior disposição física e emocional, devido aos exercícios e dinâmicas utilizados. Desse modo, os participantes demonstraram maior força e vigor para enfrentar suas barreiras. Sujeitos menos satisfeitos com o desempenho de suas habilidades reportam uma pior impressão de 
sua qualidade de vida. Todavia, se as condições negativas forem superadas pelas potencialidades do sujeito é evidente a transformação positiva em seu dia a dia (Ferreira \& Guerra, 2014; Franca, Coura, França, Basílio \& Souto, 2011).

Em comparação à população geral, indivíduos apresentam qualidade de vida inferior, já que a lesão é uma condição associada à saúde física e psicológica do sujeito e seu nível de independência, seus relacionamentos com o ambiente e suas crenças, bem como as suas relações sociais. Desse modo, a ruptura das atividades ocupacionais como o trabalho, educação, entre outros, interferem no desempenho social (Ribeiro, Monteiro \& Bártolo, 2016). Após as intervenções, foi possível identificar melhora em todos os domínios relacionados à qualidade de vida, desta forma, após a estabilização do quadro, seria recomendável que os pacientes fossem incentivados a participar de algum programa de reabilitação.

Houve melhora considerável na participação social de ambos os participantes, tal conclusão pode ser observada nos escores apresentados. Quando a pessoa com deficiência recebe suporte social apropriado, sua reorganização psicológica e processos de ajustes se dão de forma mais rápida e mais consistentes (Ferreira \& Guerra, 2014). Adultos com incapacidades que reportam bom equilíbrio emocional como resultante de um balanço entre corpo, mente e espírito, bem como harmonia com o meio, demonstram maior capacidade emocional para vencer as dificuldades apresentadas em seu dia a dia (Ribeiro, Monteiro \& Bártolo, 2016).
A saúde mental de sujeitos com lesão medular pode ser afetada pela grande dependência de outras pessoas, dificultando a adaptação e ocasionando sintomatologia ansiosa e de estresse pós-traumático, comum nessa população (Ribeiro, Monteiro \& Bártolo, 2016). No domínio saúde mental, notou-se significativa diferença nesse aspecto, denotando que as intervenções da terapia ocupacional, centradas no aprimoramento de aspectos físicos e emocionais, são efetivos na melhoria das características relacionadas à saúde mental (Silva et al., 2017).

A percepção do sujeito sobre a sua condição, aliada a aceitação de seu novo corpo, abre espaço para uma vida plena, sem dificuldades limitantes em relação aos diversos aspectos, proporcionando aos sujeitos a valorização da sua imagem corporal, capacidades e qualidade de vida (Lima, Torres \& Filho, 2014).

Sugere-se que mais estudos sejam elaborados, principalmente com um poder amostral maior, pois o pequeno número de pacientes é uma das limitações desta pesquisa.

\section{Conclusão}

A reabilitação de pacientes com lesão medular vem apresentando resultados animadores, no que tange à qualidade de vida dos sujeitos acometidos. No entanto, devemos atentar que a evidência de tais considerações, se deve a introdução de intervenções que abordam os diversos aspectos que interferem na qualidade de vida do sujeito, dentre eles, a sexualidade. Esse estudo procu- 
rou demonstrar que exercícios associados à valorização do sujeito, através da exploração das potencialidades e capacidades, por meio de aulas expositivas acerca da sexualidade humana, fortalecimento muscular específico e dinâmicas de valorização da autoestima, foram eficazes na melhoria da qualidade de vida, desempenho ocupacional e sexual dos participantes.

\section{Referências}

Abdo, C. H. N. (2006). Elaboração e validação do quociente sexual-versão masculina, uma escala para avaliar a função sexual do homem. Revista Brasileira de Medicina, 63(1-2), 42-6. Disponível em http://www.moreirajr.com.br/revistas.asp?fase $=$ r003\&id_materia $=3238$

Alves, M. L. T., \& Duarte, E. (2010). Relação entre a imagem corporal e deficiência física. Uma pesquisa bibliográfica. Revista Digital, 15 (143). Disponível em http://www.efdeportes.com/efd143/relacao-entre-a-imagem-corporal-e-deficiencia-fisica.htm

Aquarone, R., Faro, A. \& Nogueira, P. (2015). Dor neuropática central: Implicações da qualidade de vida em pacientes com lesão medular. Revista Dor, (16)4, 56-64. DOI: http://dx.doi. org/10.5935/1806-0013.20150057

Baasch, A. (2008). Sexualidade na lesão medular (Dissertação de Mestrado). Centro de Ciências da Saúde e do Esporte - UDESC, Florianópolis, Brasil.

Barbosa, V. R. (2003). A vivência da sexualidade de homens com lesão medular adquirida (Tese de Mestrado). Universidade de São Paulo, Brasil. Disponível em http://www.teses.usp.br/teses/disponiveis/59/59137/tde-03062009-142233/ pt-br.php

Borges, A.M., Brignol, P., Schoeller, S.D. \& Bonetti, A. (2012). Percepção das pessoas com lesão medular sobre a sua condição. Revista Gaúcha de Enfermagem, (33) 3, 119-125. DOI: http://dx.doi.org/10.1590/S198314472012000300016

Campos, M. F., Ribeiro A.T., Listik S., Pereira C.A., Sobrinho J. \& Rapoport A. (2008). Epidemiologia do traumatismo da coluna vertebral. Revista do Colégio Brasileiro de Cirurgiões, (35) 2, 20-27. DOI: http://dx.doi.org/10.1590/S010069912008000200005

Carleto, S. J., Cavalcanti A., Souza M., Cruz D. \& Andrade V. (2010). Estrutura da prática da terapia ocupacional: domínio e processo - $2^{\text {a }}$ edição. Revista Triângulo, (3)2, 57-147. DOI: https:// doi.org/10.18554/rt.v3i2.150

Ciconelli, R.M., Ferraz M.B., Santos, W., Meinao, I. \&.Quaresma, M.R.. (1999). Tradução para a língua portuguesa e validação do questionário genérico da avaliação de qualidade de vida SF-36. Revista Brasileira de Reumatologia, 39 (3), 143-150.

Ferreira, M. \& Guerra, M. (2014). Adaptação a lesão vertebro-medular: Adjustment to spinal cord injury. Psicologia, Saúde \& Doenças, (15) 2, 32 -40.

França, I., Coura, A., França, E., Basílio, N., \& Souto, R. (2011). Qualidade de vida de adultos com lesão medular: um estudo com WHOQOL-bref. Revista da Escola de Enfermagem da USP, 45(6), 1364-1371. DOI: 
http://dx.doi.org/10.1590/S008062342011000600013

Gonzáles, I. A. \& Sties, W. (2015). Benefícios do pilates em pacientes cardiopatas com disfunção erétil. Revista Brasileira de Medicina, (74), 15-31. Disponível em http://www.moreirajr.com.br/revistas.asp? fase $=$ r003 \&id_materia $=6082$

Kawanishi C. \& Greguol, M. (2014). Avaliação da autonomia funcional de adultos. Revista de Terapia Ocupacional da Universidade de São Paulo. (25)2, 15966. DOI: http://dx.doi.org/10.11606/ issn.2238-6149.v25i2p159-166

Law, M., Baptiste, S., Carswell, A., McCoII, M.A., Polatajko, H. \& Pollock, N. (2009). Medida Canadense de Desempenho Ocupacional (L.C. Magalhães, L.V. Magalhães \& A.A. Cardoso, org. e tradução). Belo Horizonte: UFMG. (Obra original publicada en 1990).

Lima C. G., Torres, I. P. F., \& Filho, N. R. (2014). Aspectos psicológicos associados à sexualidade do lesado medular. Estação Científica, (12), 1-7. Disponível em http://portal.estacio.br/docs\%5Crevista_estacao_cientifica/04.pdf

Maia, A.C (2012). Sexualidade depois da lesão medular: uma análise qualitativa descritiva de uma narrativa biográfica. Interação em Psicologia, 16(2). DOI: http:// dx.doi.org/10.5380/psi.v16i2.21212

Medeiros W.M., Braz, M. \& Brongholi, K. (2004). Efeitos da fisioterapia no aprimoramento da vida sexual feminina. Fisioterapia Brasil, (5) 3,188-193.

Morais, F. D., Spotti, A.R., Cohen, M.I., Mussi, S., Neto, J.S. \& Tognola, W.A. (2013). Perfil epidemiológico de pacientes com traumatismo raquimedular atendidos em hospital terciário. Coluna (12)2, 149-52. DOI: http://dx.doi.org/10.1590/S180818512013000200012

Nepomuceno, E., Melo, A.S. \& Silva, S. (2014). Alterações relacionadas aos aspectos da sexualidade no lesado medular: revisão integrativa. Revista de Enfermagem UFPE On Line, (8) 2,396-406. DOI: http://doi.org/10.5205/1981-8963v8i2a9687p396-406-2014

Ribeiro, J., Monteiro, S., Bártolo, A. (2016). Ajustamento psicossocial pós lesão vertebro- medula- associação entre eficácia e sentido na vida. Psicologia, Saúde e Doenças, 17 (3), 441-453. Disponível em http://www.redalyc.org/ pdf/362/36249164011.pdf

Rieder, M. M. (2014). Trauma raquimedular: Aspectos epidemiológicos, de recuperação funcional e de biologia molecular (Tese de doutorado). Universidade Federal do Rio Grande do Sul, Brasil. Disponível em http://www.lume.ufrgs.br/handle/10183/97212

Scussel, M.M. (2016). A fisioterapia na reabilitação sexual em pacientes com lesão medular do município de Criciúma- SC (Trabalho de Conclusão de Curso). Universidade do Extremo Sul Catarinense, Brasil. Disponível em http://repositorio. unesc.net/handle/1/335

Silva, G.A. (2015). Independência funcional da pessoa com lesão medular: do trauma a primeira internação (Tese de doutorado). Universidade Federal De Santa Catarina, Florianópolis, Brasil. Disponível em https://repositorio.ufsc.br/xmlui/ handle/123456789/158783 
Silva.C.L, Menezes, A.L.C, Cordeiro, L.M, Manzini, C.S.S, Say, K.G \& Orlandi, F.S. (2017). Qualidade de vida e sexualidade de pacientes renais crônicos em hemodiálise. Arquivos de ciências da saúde, (24)1, 67-75. DOI: https://doi. org/10.17696/2318-3691.24.1.2017.807

Tatar, Y. (2010). Body image and its relationship with exercise and sports in Turkish lower limb amputees who use prosthesis. Science \& Sport, (25), 312-317. DOI: https:// doi.org/10.1016/j.scispo.2010.02.001

Torrecilha, L.A. Costa, B.T., Lima, F.B., Santos, S.M. \& Souza, R.B. (2014). O perfil da sexualidade em homens com lesão medular. Fisioterapia em Movimento,(27)1, 39-48. DOI: http://dx.doi. org/10.1590/0103-5150.027.001.AO04 